\title{
Use of intravascular ultrasound in endovascular repair of abdominal aortic aneurysm
}

\author{
Łukasz Dzieciuchowicz ${ }^{1}$, Radosław Krzyżański ${ }^{1}$, Anita Nowak ${ }^{2}$ \\ ${ }^{1}$ Department of Vascular Surgery and Vascular Diseases, Collegium Medicum, University of Zielona Gora, Zielona Gora, Poland \\ ${ }^{2}$ Department of Vascular Surgery and Vascular Diseases, University Hospital, Zielona Gora, Poland
}

Adv Interv Cardiol 2020; 16, 2 (60): 202-205 DOI: https://doi.org/10.5114/aic.2020.96065

\section{Introduction}

Endovascular repair of abdominal aortic aneurysms (EVAR), although less invasive than surgical repair, is associated with exposure of both patients and the operating team to ionizing radiation, which may lead to the development of malignancies and eye problems [1-3]. Another disadvantage of EVAR is the use of intra-arterial iodinated contrast agents, which may lead to contrast-induced nephropathy [4]. Patients undergoing EVAR are also exposed to iodinated contrast agents during pre-interventional and post-interventional computed tomography angiography (CTA). Compared to patients who underwent open repair of the abdominal aortic aneurysm (AAA), renal function significantly deteriorated over the years in patients who underwent EVAR [5]. Thus, all techniques and strategies to reduce the exposure to radiation and contrast agents may further increase the safety of EVAR and extend its applicability. Intravascular ultrasound (IVUS) provides a real-time 360-degree image of the transverse section of the vessel. It enables precise measurement of the vessel, as well as its lumen diameter and surface. IVUS allows, to some extent, visualization of extraluminal adjacent structures within the imaging diameter. The use of a calibrated catheter allows to measure the distance between ultrasonographic anatomic vascular landmarks, such as vessel bifurcations or confluences, orifices of vascular branches and vessel crossing points, as well as between pathologic lesions such as stenosis or dilatation. Thanks to the use of automatic pull-back, it is possible to precisely measure the length of the vessel or vessel lesion. Without automatic pull-back, but using calibrated catheters, the length can also be measured using manual pull-back with an accuracy of 1 $\mathrm{cm}$. The purpose of this article is to describe the application of IVUS in EVAR.

\section{Selection of equipment and technique of intravascular ultrasound of abdominal aorta}

Two types of transducers are used in IVUS catheters, mechanical and digital. In the case of a mechanical transducer, a single element rotates at a very high speed within the catheter tip. In the case of a digital transducer, there is an electronically switched multi-element array system. The advantage of the rotational transducer is very high frequency, up to $45 \mathrm{MHz}$, which translates into very high image resolution. However, high frequency is balanced by the limited penetration depth and small imaging diameter. Therefore, in case of IVUS of aorta low frequency, digital transducers are used, such as $10 \mathrm{MHz}$ mounted on the Visions PV .035 catheter with a diameter of 8.2 Fr and compatible with 0.035-0.038" guidewires (Philips, the Netherlands, formerly Volcano, USA) with $60 \mathrm{~mm}$ imaging diameter (Figure 1). A very useful feature of this catheter is the presence of radiopaque markers: one on the scanner and 25 along the catheters at a distance of $10 \mathrm{~mm}$ from each other (Figure $2 \mathrm{~B}$ ).

The use of the Visions PV .035 catheter in the $a b$ dominal aorta requires the insertion of a $9 \mathrm{Fr}$ introducer sheath into the common femoral artery. The patient is heparinized and the Visions PV .035 catheter is advanced over the stiff wire into the ascending aorta and connected to the Volcano s5 Imaging System (Philips, the Netherlands, formerly Volcano, USA). The artifact ring is removed, and during continuous manual removal, the IVUS image is recorded. The Volcano s5 Imaging System enables immediate image analysis or pull-back recording, which can be analyzed later. During the procedure, insertion and removal of the IVUS catheter can be repeated as many times as necessary. The automatic pull-back device cannot be used during EVAR due to the length limitation.

\section{Corresponding author:}

Anita Nowak, Department of Vascular Surgery and Vascular Diseases, University Hospital, 26 Zyty St, 65-046 Zielona Gora, Poland,

e-mail: anita.nowak1994@gmail.com

Received: 10.03.2020, accepted: 17.03.2020. 

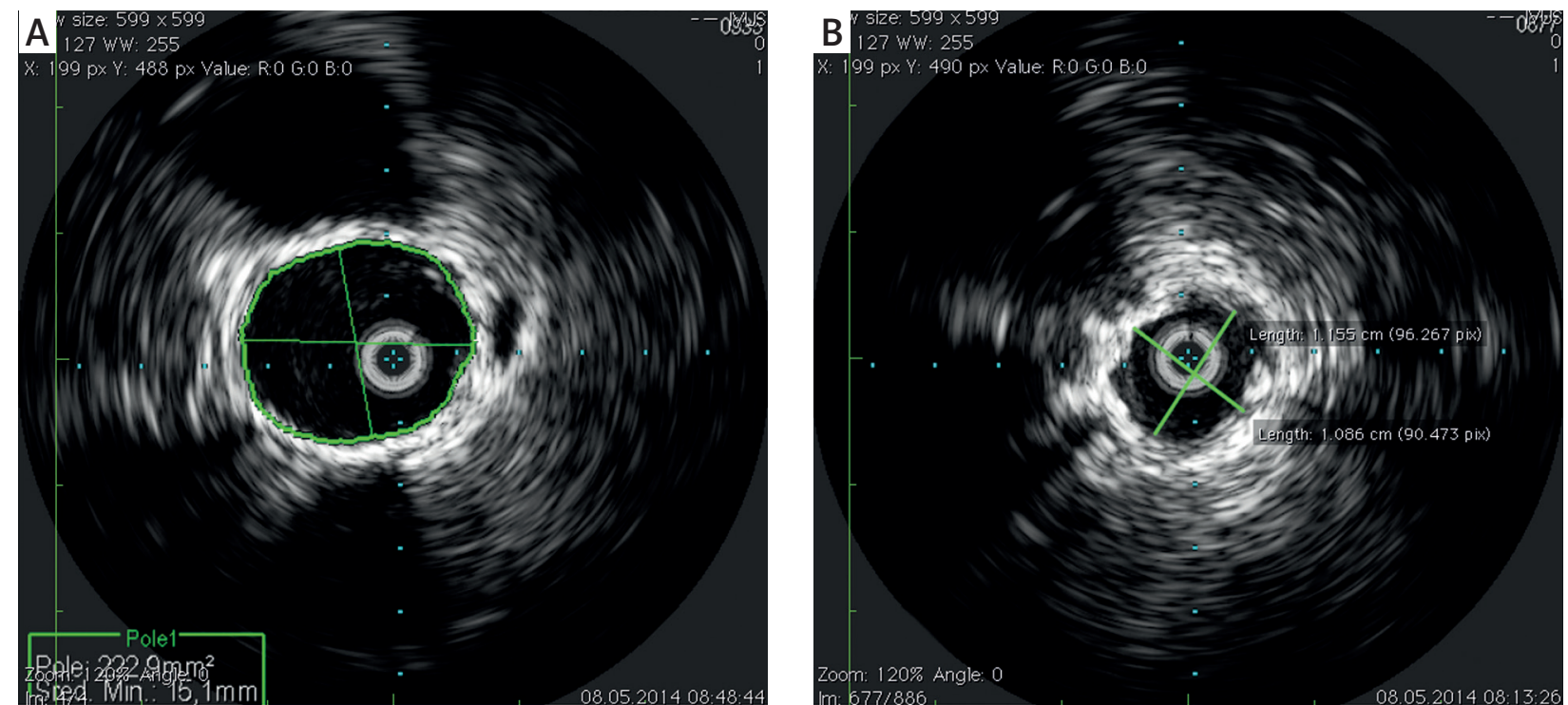

Figure 1. Semi-automatic measurement of a neck diameter of abdominal aortic aneurysm (A) and manual measurement of a diameter of common iliac artery (B)
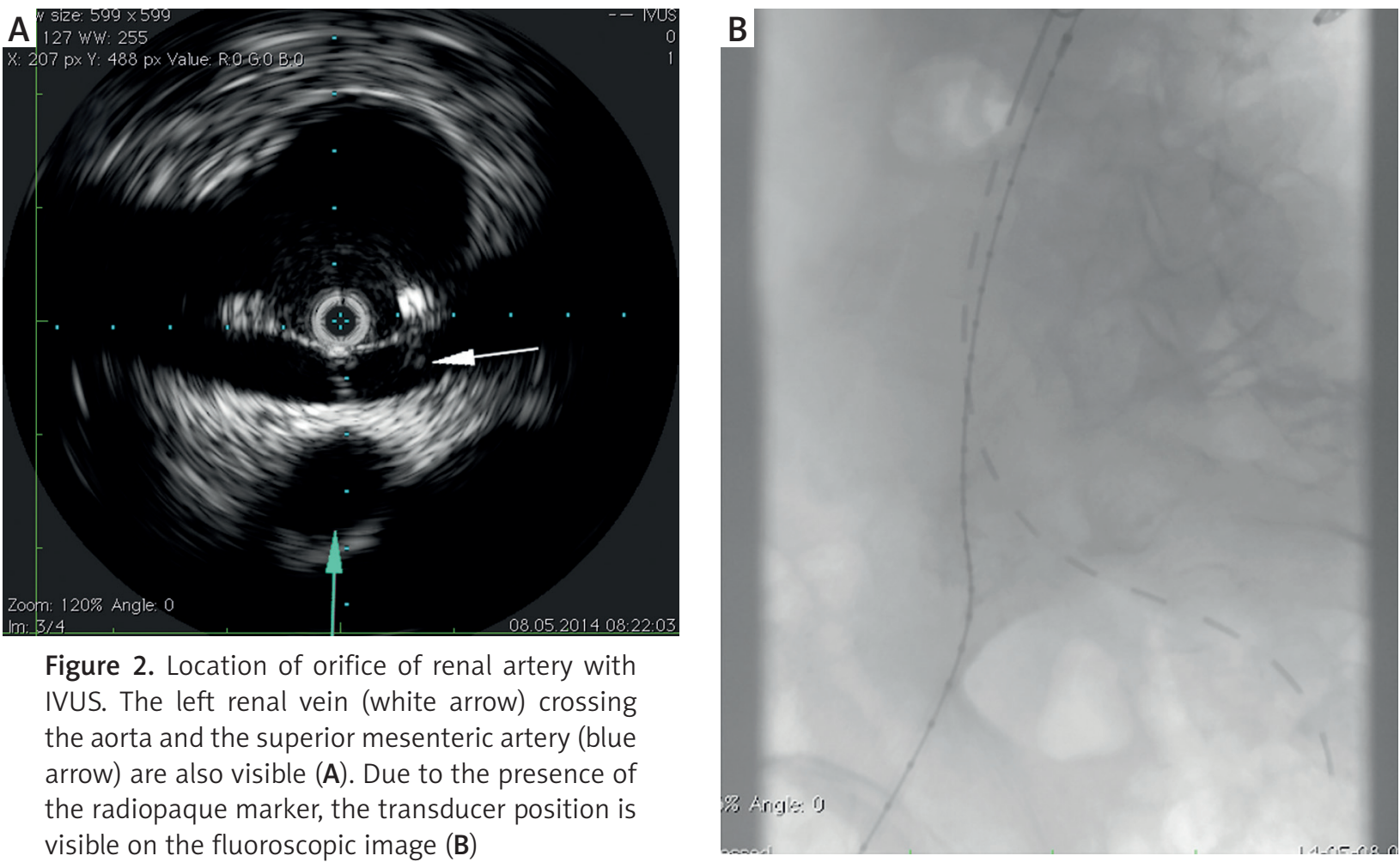

Figure 2. Location of orifice of renal artery with IVUS. The left renal vein (white arrow) crossing the aorta and the superior mesenteric artery (blue arrow) are also visible (A). Due to the presence of the radiopaque marker, the transducer position is visible on the fluoroscopic image (B)

Application of intravascular ultrasound in endovascular repair of abdominal aorta

Aneurysm sizing and graft selection

Proper aneurysm sizing and graft selection require precise measurements of the diameter and length of the aneurysm neck, length from the orifice of the lower renal artery to the bifurcations of common iliac arteries and lengths and diameters of landing zones in common or external iliac arteries. The diameters can be measured

manually, when the operator marks two points in the image and the system measures the distance between them, or semi-automatically, when the operator marks the points of the circle and the system calculates the minimum and maximum diameter, as well as the surface of the circle (Figure 1). Lengths can be measured in two ways. In the first method, once the ultrasound vascular landmark of interest is visualized, the position of the IVUS catheter tip is marked on the fluoroscopy monitor, then the IVUS catheter is moved to visualize the second 
vascular landmark of interest, and the position of the catheter tip is marked on the screen again. The distance between markings is measured using the IVUS catheter calibration. The second method does not require fluoroscopy. The marks are placed on the catheter where it enters the hemostatic valve of the introducer sheath. Then the distance between the marks is measured by IVUS catheter calibration. However, IVUS does not allow one to measure angulations, assess the morphology of the aortic branches or internal iliac arteries and plan C-arm projections for stent graft implantations. Moreover, performing IVUS of the aorta requires puncturing of femoral arteries with a $9 \mathrm{Fr}$ introducer sheath. Therefore, IVUS is not routinely used for EVAR planning. It may be an alternative to initial catheter angiography for those who perform it before introducing the delivery system into the abdominal aorta.

\section{Guidance for stent graft main body deployment}

The IVUS catheter is advanced over the guidewire from the contralateral side to the insertion of the main body of the stent graft. The orifice of the lower renal artery is localized by IVUS, and the transducer is maintained at this level, while the radiopaque marker of the transducer is visible on the fluoroscopy monitor and serves as a marker for the stent graft deployment (Figure 2). The advantage of IVUS over fluoroscopy is real-time imaging, which eliminates the need for repetitive angiograms to control the deployment of the main body of the stent graft. However, this can be hindered if the delivery system of the graft is positioned between the orifice of the lower renal artery and the IVUS transducer.

\section{Confirmation of successful catheterization of contralateral main body gate}

The contralateral limb catheterization needs to be performed under fluoroscopic control, but IVUS can be used to check the spatial orientation of the contralateral limb. Limb catheterization should always be confirmed, which is usually done by introducing the pigtail catheter within main body of the stent graft. With IVUS, the proper position of the guidewire within the sten graft limb can be confirmed with $100 \%$ accuracy and without the use of fluoroscopy.

\section{Selection of length and deployment of iliac extensions}

The ultimate selection of the length of iliac extensions in a standard procedure requires oblique projection arteriography, which increases radiation exposure. With IVUS, the distance between the edges of the main body limbs and the bifurcations of common iliac arteries can be measured as described above. The position of the IVUS transducer in the iliac bifurcation can be marked on the fluoroscopy screen and guide the deployment of the iliac extensions of the stent graft.

\section{Post-deployment assessment}

After complete implantation of the stent graft, IVUS can be used to confirm the adequate position of the stent graft in relation to renal arteries and the apposition of the stent graft in landing zones in the aneurysm neck and iliac arteries. Lack of proper apposition of the stent graft in landing zones, especially in the presence of post-deployment endoleak, would indicate the need for further expansion of a segment of the stent graft with a semi-compliant balloon or other adjunctive procedures. The possibility of confirming the apposition of the stent graft is a great advantage of IVUS over plain catheter angiography and is an alternative to $3 \mathrm{D}$ rotational angiography that would expose the patient and the operating team to an increased dose of ionizing radiation. IVUS in this indication would be even more useful in the case of a fenestrated or branched stent graft, when the radiation dose is much higher. IVUS can also visualize the presence of kinking or stenosis in the iliac extension that would require correction with balloon angioplasty and stenting to prevent occlusion of the stent graft limb.

\section{IVUS limitation in EVAR}

One of the limitations is the lack of flow visualization with the use of Visions PV 0.035 catheters, which do not allow to detect the presence of endoleaks. Although an intravascular ultrasound probe with Doppler and color Doppler capabilities and appropriate imaging range is currently available on the market (AcuNav; Siemens, Mountain View, USA), it also has some limitations [6]. AcuNav is a vector phased-array probe that visualizes only one fourth of the circle and prevents accurate measurement of vessel diameter. Another drawback that probably limits the widespread use of IVUS during stent graft implantation is the cost of the IVUS catheter, which is even higher for a vector phased-array probe.

\section{Conclusions}

IVUS has a potential to decrease radiation exposure and contrast volume during endovascular repair of abdominal aortic aneurysm. This should be confirmed in clinical trials.

\section{Conflict of interest}

The authors declare no conflict of interest.

\section{References}

1. Greenhalgh RM, Brown LC, Kwong GP, et al. EVAR trial participants. Comparison of endovascular aneurysm repair with open repair in patients with abdominal aortic aneurysm (EVAR trial 1), 30-day operative mortality results: randomised controlled trial. Lancet 2004; 364: 843-8. 
2. Rajaraman P, Doody MM, Yu CL, et al. Cancer Risks in U.S. Radiologic Technologists Working With Fluoroscopically Guided Interventional Procedures, 1994-2008. AJR Am J Roentgenol 2016; 206: 1101-8.

3. Roguin A, Goldstein J, Bar O, et al. Brain and neck tumors among physicians performing interventional procedures. Am J Cardiol 2013; 111: 1368-72.

4. Saratzis A, Melas N, Mahmood A, et al. Incidence of acute kidney injury (AKI) after endovascular abdominal aortic aneurysm repair (EVAR) and impact on outcome. Eur J Vasc Endovasc Surg 2015; 49: 534-40.

5. Al Adas Z, Shepard AD, Nypaver TJ, et al. Long-term decline in renal function is more significant after endovascular repair of infrarenal abdominal aortic aneurysms. J Vasc Surg 2018; 68: 739-48.

6. Eriksson MO, Wanhainen A, Nyman R. Intravascular ultrasound with a vector phased-array probe (acunav) is feasible in endovascular abdominal aortic aneurysm repair. Acta Radiol 2009; 50: 870-5. 